\title{
Better Bounds for Coalescing-Branching Random Walks
}

\author{
MICHAEL MITZENMACHER, Harvard University \\ RAJMOHAN RAJARAMAN, Northeastern University \\ SCOTT ROCHE, Akamai Technologies
}

Coalescing-branching random walks, or cobra walks for short, are a natural variant of random walks on graphs that can model the spread of disease through contacts or the spread of information in networks. In a $k$-cobra walk, at each timestep, a subset of the vertices are active; each active vertex chooses $k$ random neighbors (sampled independently and uniformly with replacement) that become active at the next step, and these are the only active vertices at the next step. A natural quantity to study for cobra walks is the cover time, which corresponds to the expected time when all nodes have become infected or received the disseminated information.

In this article, we extend previous results for cobra walks in multiple ways. We show that the cover time for the 2-cobra walk on $[0, n]^{d}$ is $O(n)$ (where the order notation hides constant factors that depend on $d$ ); previous work had shown the cover time was $O(n \cdot \operatorname{polylog}(n))$. We show that the cover time for a 2-cobra walk on an $n$-vertex $d$-regular graph with conductance $\phi_{G}$ is $O\left(d^{4} \phi_{G}^{-2} \log ^{2} n\right)$, significantly generalizing a previous result that held only for expander graphs with sufficiently high expansion. And, finally, we show that the cover time for a 2-cobra walk on a graph with $n$ vertices and $m$ edges is always $O\left(m n^{3 / 4} \log n\right)$; this is the first result showing that the bound of $\Theta\left(n^{3}\right)$ for the worst-case cover time for random walks can be beaten using 2-cobra walks.

CCS Concepts: - Mathematics of computing $\rightarrow$ Graph algorithms; Probabilistic algorithms; Stochastic processes;

Additional Key Words and Phrases: Random walks, networks, information spreading, cover time, epidemic processes

\section{ACM Reference format:}

Michael Mitzenmacher, Rajmohan Rajaraman, and Scott Roche. 2018. Better Bounds for Coalescing-Branching Random Walks. ACM Trans. Parallel Comput. 5, 1, Article 2 (June 2018), 23 pages.

https://doi.org/10.1145/3209688

\section{INTRODUCTION}

Random walks provide a fundamental mathematical model for many basic network processes. In disease models, transmission of a virus can be modeled by the virus moving according to a random walk on a graph representing a human contact network; computer viruses can be modeled

M.M. was supported in part by NSF Grants No. CNS-1228598, No. CCF-1320231, and No. CCF-1535795. R.R. and S.R. were supported in part by NSF CCF-1422715, NSF CCF-1535929, and an ONR grant on network algorithms.

Authors' addresses: M. Mitzenmacher, School of Engineering and Applied Sciences, Harvard University, 33 Oxford Street, Cambridge, MA 02138; email: michaelm@eecs.harvard.edu; R. Rajaraman, 202 WVH, College of Computer and Information Science, Northeastern University, Boston MA 02115; email: rraj@ccs.neu.edu; S. Roche, Akamai Technologies, 150 Broadway, Cambridge MA 02142; email: str7@cornell.edu.

Permission to make digital or hard copies of all or part of this work for personal or classroom use is granted without fee provided that copies are not made or distributed for profit or commercial advantage and that copies bear this notice and the full citation on the first page. Copyrights for components of this work owned by others than ACM must be honored. Abstracting with credit is permitted. To copy otherwise, or republish, to post on servers or to redistribute to lists, requires prior specific permission and/or a fee. Request permissions from permissions@acm.org.

(C) 2018 ACM 2329-4949/2018/06-ART2 $\$ 15.00$

https://doi.org/10.1145/3209688

ACM Transactions on Parallel Computing, Vol. 5, No. 1, Article 2. Publication date: June 2018. 
similarly $[15,20,31]$. Variants of random walks can also be used for information dissemination, using message-passing protocols where a message is passed from neighbor to neighbor via a random walk $[9,19]$. Such protocols require little state information and are robust to various types of faults, and are therefore useful in many distributed networks [4]. More generally, random walks provide a fundamental primitive for network algorithms for information propagation, search, routing, and load balancing.

In many of these settings, a central measure of interest is the cover time, the expected time for the random walk to cover all of the vertices of the underlying network. In disease models, this corresponds to the time until all vertices in the network have been exposed to the virus; in messagepassing protocols, this corresponds to the time until all vertices have received the message.

Parallel random walks provide a natural generalization of standard random walks, with multiple random walks traversing the network simultaneously, and several articles have analyzed the performance of parallel random walks (as we describe in the related work section). A related variant, less well-studied and understood, are coalescing-branching random walks, or cobra walks for short [15]. In a cobra walk, at each timestep, a subset of the vertices are active; typically, in the initial state, a single vertex would be active. At each timestep, each active vertex chooses $k$ random neighbors (sampled independently and uniformly with replacement) that become active at the next step. A vertex is active at step $t$ if and only if it was chosen by an active vertex in the previous step. When $k>1$, each walk branches at that step into multiple walks, but multiple walks then coalesce when they reach the same vertex at the same time. We refer to a cobra walk where, at each timestep, a vertex chooses $k$ active neighbors as a $k$-cobra walk for convenience. (One could further study variations where the branching varied based on the vertex or the timestep, or was governed by a random distribution; we do not do that here.)

As examples of cobra walks, in the message passing setting, a $k$-cobra walk corresponds to a network where a vertex may send $k$ outgoing copies of the message to neighbors during a timestep instead of just one. In disease networks, a cobra walk corresponds to an idealized process within the Susceptible Infected Susceptible model (or SIS model): in each timestep, an infected agent infects $k$ random neighbors and recovers, but can be infected again (including at the next timestep).

In contrast to results in parallel random walks, where the number of walks is a parameter, in cobra walks the number of active vertices varies over time and its behavior depends significantly on the network. One might expect cobra walks to yield significant improvements in the cover time over standard random walks, based on their power to reproduce, even if limited by coalescence. The goal of our article is to formally and theoretically bound the performance of cobra walks, focusing on the cover time. While our results have potential applications to distributed protocols and disease models, as suggested above, we also believe that $k$-cobra walks are a natural mathematical model worthy of study in their own right.

\subsection{Our Results and Techniques}

We are motivated by the prior work [15], which obtained bounds on the cover time of cobra walks on trees, grids, and expanders. Our article pushes those results further, in several directions. Our primary results are the following:

-We show that the cover time for the 2-cobra walk on $[0, n]^{d}$ is $O(n)$, where the constant in the order notation can depend on $d$. This improves on the previous bound of $O(n \cdot \operatorname{polylog}(n))$ [15]. With respect to $n$, our result is optimal.

- We show that the cover time for the 2-cobra walk for a $d$-regular graph with conductance $\phi_{G}$ is $O\left(d^{4} \phi_{G}^{-2} \log ^{2} n\right)$. This generalizes a similar result in Reference [15] for expander graphs 
with sufficiently high expansion. Our new result holds for any $d$-regular graph, and expresses the bound as a function of the conductance.

-We provide a result for general graphs, showing that the cover time for a 2-cobra walk is always $O\left(m n^{3 / 4} \log n\right)$. For standard random walks, there are graphs where the cover time is $\Theta\left(n^{3}\right)$. This is the first result showing that cobra walks can beat the corresponding worstcase bound for random walks. We also establish an $O\left(n^{2-1 / d} \log n\right)$ upper bound on the cover time for arbitrary $d$-regular graphs, again improving the tight quadratic bound for standard random walks.

Our main techniques involve making use of the parallelism inherent in cobra walks, and by thinking of cobra walks as a union of biased random walks. In some settings, we can show the cobra walk goes through an initial phase that instantiates a large number of essentially parallel random walks, and then analyze the behavior of these random walks. Here we have to take care of the dependency challenges introduced by coalescing, as random walks can essentially disappear when several collide at a vertex. In other settings, we think of our cobra walk as being a single walk moving toward a specific vertex, and then eventually taking a union bound over all vertices. At each step, we can choose to follow the active vertex that moves toward the target vertex and discard the others. This approach simplifies the analysis by allowing us to focus on a single walk, where now the $k$ choices correspond to a bias in the walk that we can model. The downside of such an analysis, however, is that it does not take full advantage of the power of parallelism inherent to cobra walks.

We note that in independent work published in parallel with the conference version of this article, Cooper et al. [12] presented an alternative analysis of cobra walks using a novel duality relation between cobra walks and a different discrete epidemic process. They bounded the cover time on regular graphs in terms of the second smallest eigenvalue $\lambda$ of the transition matrix of the graph: the cover time is $O(\log n)$ if $1-\lambda$ is greater than a positive constant (as is the case for an expander graph), and $O\left((\log n) /(1-\lambda)^{3}\right)$ if $1-\lambda$ is significantly greater than $\sqrt{(\log n) / n}$. The bound achieved by Reference [12] for expander graphs is optimal; for general graphs, our bounds and theirs are incomparable. In more recent results obtained subsequent to this article, Cooper et al. [13] have obtained an improved $O\left(n^{2} \log n\right)$ bound for cobra walks for general graphs and an improved bound for regular graphs in terms of the second eigenvalue of the graph.

\subsection{Background and Related Work}

The cobra walk process has structural similarities with several other fairly diverse stochastic processes: branching processes, gossip protocols, and random walks (including parallel random walks, coalescing random walks, and other variants). Despite these commonalities, cobra walks resist being fully described by any of these other processes; furthermore, analysis techniques used for these other processes often have no clear use or power in analyzing cobra walks.

Branching and Coalescing Processes. Branching processes appear in many disciplines, from nuclear physics to population genetics, on various discrete and continuous structures [6, 21, 24]. Another related topic is the study of coalescing processes and voter models (see, for example Reference [11]). Naturally, there is also work on processes that contain both branching and coalescing elements [3,29], although, unlike our work, these analyses tend to operate in continuous time and on either restricted topologies or infinite spaces. The restriction of cobra walks to discrete time effectively disallows the use of differential-equation-based analysis that often yields results in continuous-time processes (see, for example, References [20, 22, 31]). 
Gossip and Rumor-Spreading Mechanisms. Gossip-based algorithms have been used successfully to design efficient distributed algorithms for a variety of problems in networks such as information dissemination, aggregate computation, constructing overlay topologies, and database synchronization (e.g., see Reference [9] and the references therein). There are three major variants of gossip-based processes: push-based models, in which the members of the set of informed vertices each select a neighbor and inform that neighbor (if it is not already informed), pull-based models, in which uninformed vertices select neighbors and poll them for information, and push-pull, which is a combination of the first two.

Cobra walks bear the closest resemblance to push-based gossip models. Indeed, Reference [19] shows that the push process completes in every undirected graph in $O(n \log n)$ steps with high probability, and this bound has been conjectured to hold for cobra walks [15]. However, the similarity between the two is in many ways superficial. If we view a gossip process as a Markov chain on the state space of the set of all subsets of vertices (representing the sets of possible informed vertices), this Markov chain has a single absorbing state (assuming the graph is connected) in which every vertex is informed. On the other hand, performing a similar projection of a cobra walk onto a Markov chain of the $2^{n}$ possible subsets of vertices that could be active at any time, we see that there is no absorbing state and with the addition of self-loops, the chain can be made ergodic.

Random Walks and Parallel Random Walks. Cobra walks also resemble standard random walks and parallel variants. For simple random walks, the now classic work of Feige $[17,18]$ showed that the cover time on any graph lies between $\Theta(\log n)$ and $O\left(n^{3}\right)$. A formal model of biased random walks was introduced in Reference [5] with the motivation of studying imperfect sources of randomness. Specifically, these biased walks allow a controller to fix, at each step of the walk, the next step with a small probability, with the aim of increasing the stationary probability at a target set of vertices. A variant of the biased walk of Reference [5] plays a significant role in our analysis of cobra walks for general graphs.

Additional work has considered speeding up the cover time by modifying the underlying process. Adler et al. [1] studied a process on the hypercube in which, in each round, a vertex is chosen uniformly at random and covered; if the chosen vertex was already covered, then an uncovered neighbor of the vertex is chosen uniformly at random and covered. For any $d$-regular graph, Dimitrov and Plaxton showed that a similar process achieves a cover time of $O(n+(n \log n) / d)$ [14]. For expander graphs, Berenbrink et al. showed a simple variant of the standard random walk that achieves a linear (i.e., $O(n))$ cover time [7].

Parallel random walks, first studied in Reference [8] for the special case where the starting vertices are drawn from the stationary distribution and in Reference [2] for arbitrary starting vertices, also appear related to cobra walks. Nearly tight results on the speedup of cover time as a function of the number of parallel walks have been obtained by Reference [16] for several graph classes including the cycle, $d$-dimensional meshes, hypercube, and expanders. However, again the similarity is somewhat superficial. A parallel random walk with $k$ independent walks can be mapped to an undirected random walk on a graph known as the tensor product. As such, much of the machinery of the analysis of simple random walks can be applied to the parallel case. Applying a similar approach to a cobra walk is not feasible, although for cobra walks one can convert the tensor product into a directed graph, changing the topology significantly. As such, generally, the techniques that can be used for parallel random walks cannot be used directly for the cobra walk. Indeed, one can view the dependencies on the locations of the other active nodes in a cobra walk as a manifestation of this difference. Cobra walks suffer from the "time's arrow" effect: locally, most individual steps are reversible, but as a cobra walk expands, the likelihood that it will coalesce back to a single vertex grows exponentially unlikely. Despite these difficulties, 
the tensor product graph can be useful when studying the movement of a small number of pebbles in a cobra walk, and we make use of this technique in obtaining a general bound based on conductance.

\section{PRELIMINARIES}

Let $G$ be a connected graph with vertex set $V$ and edge set $E$, and let $|V|=n$, except for the case when we are analyzing the grid, in which case we let $V=[0, N]^{d}$. A $k$-coalescing-branching $(k$ cobra) walk is defined as follows: It starts at time $t=0$ at an arbitrary vertex $v$, at which a pebble is placed. In the next and every subsequent timestep, every pebble in $G$ clones itself $k-1$ times (so that there are now $k$ indistinguishable pebbles at each vertex that originally had a pebble). Each pebble then independently selects a neighbor of its current vertex uniformly at random and moves to it. Once all pebbles have made their moves, the coalescing phase begins: if two or more pebbles are at the same vertex they coalesce into a single pebble, and the next round begins.

For timestep $t, S_{t}$ is the active set, which is the set of all vertices of $G$ that have a pebble. Define the cover time of a cobra walk to be the maximum over all vertices $v$ of the expectation of the minimum time $T$, at which all vertices have belonged to some $S_{t}$ for $t \leq T$ when the cobra walk is started at $v$. We note that while our results are stated as bounds on the cover time, all of the results in this article actually give bounds on the time to cover all the vertices in the graph with high probability, as is clear from the proofs. Hence we may also refer to the time at which all vertices have been covered, where the meaning is clear. The hitting time $H(u, v)$ is the expectation of the minimum time it takes for any pebble originating from a cobra walk that starts at $u$ to reach $v$. The maximum hitting time $h_{\max }$ is $\max _{u, v \in V} H(u, v)$.

We make use of an extension of Matthews' Theorem, which relates the cover time of a random walk to the maximum hitting time. The following theorem was proven in Reference [15]:

Theorem 2.1. Let $G$ be a connected graph on $n$ vertices. Let $W$ be a cobra walk on $G$ starting at an arbitrary vertex. Then the cover time of $W$ on $G$ is bounded above by $O\left(h_{\max } \log n\right)$; in fact, $W$ covers all of $G$ in $O\left(h_{\max } \log n\right)$ steps with high probability.

Finally, we make use of a combinatorial property of the graph, the conductance. Define the conductance of a set $S \subseteq V$ as $\phi(S)=|\partial(S)| / \operatorname{vol}(S)$, where $\partial(S)=\sum_{(u, v): u \in S, v \notin S} 1$ and $\operatorname{vol}(S)=$ $\sum_{u \in S} d(u)$. Then the conductance $\Phi_{G}$ of the graph is $\min _{S: \operatorname{vol}(S) \leq \operatorname{vol}(V) / 2} \phi(S)$. For the purposes of this article, we say that a $d$-regular graph is an $\epsilon$-expander if the conductance of the graph is greater than or equal to $\epsilon$.

\section{TIGHT RESULTS FOR GRIDS}

We show that the cover time for the $d$-dimensional grid using a 2-cobra walk $[0, N]^{d}$ is $O(N)$, where the order notation hides constant factors and other terms that depend on $d$; indeed, we show all vertices are covered in $O(N)$ steps with high probability. Previous work has shown that the cover time is $O(N \cdot \operatorname{polylog}(N))$ [15]. We note that the results of Reference [15] use a slightly different notation, working with $n$ total nodes, or $\left[0, n^{1 / d}-1\right]^{d}$. We have opted to work over $[0, N]^{d}$ for convenience, with conversion factor $N=n^{1 / d}$. Our result is clearly tight in its dependence on $N$. Moreover, it shows that in some circumstances one can avoid using tools such as Matthews' Theorem, which had been used previously in this setting [15], and necessarily adds in an additional logarithmic factor in the number of vertices over the hitting time.

The case of $d=2$ is simple and instructive; we sketch a proof, but do not go into full detail as we have a more detailed proof for the general case.

Lemma 3.1. The 2-cobra walk on $[0, N]^{2}$ has cover time $O(N)$. 
Proof. Let $v_{0}$ be the starting vertex of the walk. We show that with high probability all vertices are reached within $O(N)$ steps, and the result follows, because if every vertex is hit within $T$ steps with probability $p$, the expected time to cover all vertices is bounded above by $T / p$.

Let $v_{1}=\left(x_{1}, y_{1}\right)$ be some other vertex on the grid. Let $X_{t}$ be the Manhattan distance between the closest pebble of the cobra walk and $v_{1}$ after $t$ steps, which we will refer to as time $t$. (All distances in this section will refer to Manhattan distances.) Let $u_{t}=\left(a_{t}, b_{t}\right)$ be some arbitrary vertex with a pebble of distance $X_{t}$ from $v_{1}$ at time $t$. We show by cases that there is drift, so that the expectation of $X_{t}$ decreases linearly over time, even when at each step we pessimistically consider only the single vertex $u_{t}$ and not other additional pebbles; it follows from standard results in random walks $v_{1}$ is reached after $O(N)$ steps with probability $1-O\left(1 / N^{3}\right)$. Hence all vertices are covered after $O(N)$ steps with probability $1-O(1 / N)$, and the result follows.

If $x_{1} \neq a_{t}$ and $y_{1} \neq b_{t}$, the probability that at least one of the two pebbles at $u_{t}$ moves closer to $v_{1}$ is at least $1-(1 / 2)^{2}=3 / 4$, since each pebble moves closer to $v_{1}$ with probability at least $1 / 2$. (It can be more if a pebble $v_{1}$ is on the boundary of a grid.) If either $x_{1}=a_{t}$ or $y_{1}=b_{t}$ but not both, the probability that at least one of the two pebbles at $u_{t}$ moves closer to $v_{1}$ could be as small as $1-(3 / 4)^{2}=7 / 16<1 / 2$, since each pebble could move closer to $v_{1}$ with probability only $1 / 4$. (This probability would be $1-(2 / 3)^{2}=5 / 9$ if for example $x_{1}=a_{t}=n$, but off the grid boundary this is not the case.) Over one step, then, the expected distance may be increasing. So we instead consider two steps. It is important to note that if the distance increases on the first of the two steps but (at least) one pebble moves so that $x_{1} \neq a_{t}$ and $y_{1} \neq b_{t}$ after the first step, it improves our probability that the distance decreases in the second step. We find taking cases, assuming that $u_{t}$ is at least distance 2 from the boundary and from $v_{1}$, that $X_{t}$ increases by 2 by with probability $\frac{1}{16} \frac{9}{16}+\frac{1}{2} \frac{1}{4}=\frac{41}{256}$. $X_{t}$ decreases by 2 by with probability $\frac{7}{16} \frac{7}{16}=\frac{49}{256}$. (Similar (better) results can be shown when $u_{t}$ is near a boundary.)

We therefore see that $X_{t}$ has negative drift (except at $X_{t}=1$, where the drift is slightly positive), and therefore the time to reach 0 can be shown to be $O(N)$ with probability $1-O\left(1 / N^{3}\right)$ as claimed.

The analysis above suggests technical difficulties to overcome with a direct approach for general $d$; the behavior is slightly different at the boundaries of the grid, and when coordinates match in one or more dimensions, it makes analyzing the drift more difficult. The analysis makes clear that for any fixed $d$, there should be a large enough constant value of $k$ so that the cover time for the $k$-cobra walk is $O(N)$ on the $d$-dimensional grid $[0, N]^{d}$. One just needs a large enough value of $k$ so that the pebble nearest a target vertex drifts toward that vertex.

We actually show the stronger result that the 2-cobra walk has cover time $O(N)$ on the $d$ dimensional grid $[0, N]^{d}$, where the order notation hides constants that depend on $d$. We prove this below; we have not aimed to optimize the constant factors. The intuition for the proof is the following. If we look at the distance between the closest point on the cobra walk and our target vertex in any single dimension, it behaves like a biased random walk, with a bias toward 0 . Hence, after $O(N)$ steps, each individual dimension has matched coordinates, with high probability. Indeed, if each chain was an independent biased random walk with constant bias (independent of $d$ ), after $O(d N)$ steps we would expect each independent walk to be near its stationary distribution, in which case each chain would be at 0 with some constant probability $\gamma$, and hence it would take roughly $\gamma^{d}$ steps for all chains to be at 0 simultaneously. Sadly, as usual, the fact that the chains are not themselves independent causes significant technical challenges.

Theorem 3.2. The 2-cobra walk has cover time $O(N)$ on the d-dimensional grid $[0, N]^{d}$ for any constant $d$. 
Proof. We break the proof into steps. As in Lemma 3.1, we consider the distance to a target vertex over all dimensions for some pebble generated by the 2-cobra walk. We pessimistically keep track of only a single pebble. Specifically, our state at time $t$ can be defined as follows. Let $\left(z_{1,0}, z_{2,0}, \ldots, z_{d, 0}\right)$ be such that the distance from the initial pebble to the target vertex is $z_{i, 0}$ for the $i$ th grid dimension. More generally, assume there is some pebble at the $t$ th step so that $\left(z_{1, t}, z_{2, t}, \ldots, z_{d, t}\right)$ gives the distance from that pebble to the target vertex in each dimension. We update the $z_{i, t}$ values over timesteps as follows. If our two choices of pebbles generated from that pebble move in the same dimension, we choose the pebble that moves closer to the target, if such a pebble exists. If our two choices of pebbles generated from that pebble move in different dimensions $i$ and $j$, there are several cases. If $z_{i, t}=0$ but $z_{j, t} \neq 0$, we choose the pebble that moves in dimension $j$. If $z_{i, t}=0$ and $z_{j, t}=0$, we choose the pebble randomly. If $z_{i, t} \neq 0$ and $z_{j, t} \neq 0$, if both choices of pebbles move closer or both move farther away from the target, we choose the pebble randomly; otherwise we choose the pebble that moves closer.

Lemma 3.3. In each dimension, we have that if $z_{i, t} \neq 0$, then $z_{i, t}$ changes in the next step with probability at least $1 /(2 d-1)$, and conditioned on the ith dimension being the value that changes, it decreases with probability at least $1 / 2+1 /(8 d-4)$. If $z_{i, t}=0$, it increases in the next step with probability at most $2 /(d+1)$.

Proof. This follows directly from the description above. We note that worst case with regard to the bias is when one dimension has $z_{i, t} \neq 0$ and $z_{j, t} \neq 0$ for $j \neq i$. In this case, the only bias that favors the $i$ th dimension decreasing rather than increasing stems for both choices being in the $i$ th dimension. With probability $2(d-1) / d^{2}$ the pebble is chosen to move in the $i$ th dimension and some other dimension, in which case the pebble is equally likely to move closer or further to the target. With probability $1 / d^{2}$ dimension $i$ is chosen for both moves, in which case it moves closer with probability $3 / 4$. Hence, conditioned on $z_{i, t}$ changing, it increases with probability

$$
\frac{(d-1) / d^{2}+(3 / 4) / d^{2}}{2(d-1) / d^{2}+1 / d^{2}}=\frac{d-1 / 4}{2 d-1}=\frac{1}{2}+\frac{1}{8 d-4}
$$

as claimed.

With regard to which dimension moves, we notice that when $z_{i, t} \neq 0$, the probability of moving is least when we are on the boundary in the $i$ th dimension (and hence there is just one move in that dimension), and other dimensions are not. When $z_{i, t}=0$, that dimension is most likely to move if all others are on the boundary. The above bounds reflect these cases.

Our multi-dimensional biased random walk has a natural interpretation as a discrete time queueing system, where customers arrive and wait at a randomly chosen queue, where the arrival rate is slightly smaller than the departure rate (except when a queue is empty). In this setting, our question concerns the time until the system empties from a given starting state. Surprisingly, despite this connection, we could not find a statement corresponding to our desired result in the literature.

The following follows easily from the bias shown above.

LEMmA 3.4. In each individual dimension, if the $z_{i, t}$ are bounded above by $N$, then $z_{i, t}=0$ for $t=O\left(d^{2} N\right)$ with probability $1-O\left(1 / N^{d+1}\right)$.

Proof. Let $X$ be the number of steps taken in the $i$ th dimension over the first $64 d^{2} N$ steps. Then by Lemma 3.3, $\mathbb{E}[X]>32 d N$, and using a Chernoff bound (e.g., Reference [26, Exercise 4.7])

$$
\operatorname{Pr}(X \leq 16 d N) \leq(e / 2)^{-8 d N} .
$$

The expected difference between the number of steps that decrease $z_{i, t}$ and the number of steps that increase $z_{i, t}$ grows with $X$, so we pessimistically condition on $X \geq 16 d N$. Suppose that in this 
case that 0 is never reached, so the bias remains in effect over all $16 d N$ steps. Let $Y$ be number of decreases in the first $16 d N$ steps. Then $\mathbb{E}[Y] \geq 8 d N+2 N$, and again using a Chernoff bound (e.g., Reference [26, Exercise 4.7])

$$
\operatorname{Pr}(Y \leq 8 d N+N \mid X \geq 16 d N) \leq\left(\frac{e^{-\delta}}{(1-\delta)^{(1-\delta)}}\right)^{-8 d N+2 N},
$$

where $\delta=1 /(8 d+2)$. Notice that if $Y \geq 8 d N+N$ then in fact 0 was reached. Hence the total probability that 0 is not reached is bounded above by

$$
\operatorname{Pr}(Y \leq 8 d N+N \mid X \geq 16 d N)+\operatorname{Pr}(X \leq 16 d N) \leq(e / 2)^{-8 d N}+\left(\frac{e^{-\delta}}{(1-\delta)^{(1-\delta)}}\right)^{-8 d N+2 N},
$$

which is clearly $O\left(1 / N^{d+1}\right)$.

Similarly, the following result is standard for biased random walks.

LEMMA 3.5. Once $z_{i, t}$ hits 0 , with probability $1-O\left(1 / N^{d+2}\right)$, it will remain below $c_{d} \ln N$ for some constant $c_{d}$ (depending on $d$ ) over the next $100 d^{2} N^{2}$ steps.

Proof. We may pessimistically assume that all $N^{2}$ steps are performed in the $i$ th dimension. By the natural coupling we have that the probability that $z_{i, t}$ reaches $c_{d} \ln N$ in exactly $k$ steps after it hits 0 is less than the probability in equilibrium that a biased random walk with probability $\frac{1}{2}+\frac{1}{8 d-4}$ is at $c_{d} \ln N$. (Technically, the biased random walk doesn't have an equilibrium distribution, because of parity; it will be an even number of steps from its starting point after an even number of steps. We can add an arbitrarily small self-loop probability and increase the number of steps accordingly; we use $N^{2}$ steps and assume the appropriate equilibrium distribution for convenience.) The equilibrium distribution for this biased random walk, where $\pi_{j}$ is the probability of being at $j$ in equilibrium, is easily found by detailed balance equations, which yield

$$
\pi_{j}=\frac{2}{4 d-1}\left(\frac{4 d-3}{4 d-1}\right)^{k}
$$

As $\pi_{j}$ is geometrically decreasing, for $j=c_{d} \ln n$ for some constant $c_{d}$, we have $\pi_{k}$ will be less than $1 /\left(100 d^{2} N^{d+4}\right)$, and hence over the $100 d^{2} N^{2}$ timesteps we see $z_{i, t}$ never reaches $c_{d} \ln N$ with probability $1-O\left(1 / N^{d+2}\right)$.

We now use the following lemma, which in a slightly different form appears in Theorem 7 of Reference [15]. We sketch the proof for completeness.

LEMMA 3.6. Starting from $\left(z_{1, t}, z_{2, t}, \ldots, z_{d, t}\right)$, where each $z_{i, t}$ is at most $c_{d} \ln N$, with probability $\Omega\left(1 /(\ln \ln N)^{c}\right)$ for some constant $c$, there is a $u=\alpha \ln N$ such that for some $k \leq u, z_{i, t+k}=0$ for all $i=1, \ldots, d$.

Proof. The analysis is broken into phases. The first phase is of length $O(\ln N)$, the second phase is of length $O\left((\ln N)^{1 / 2}\right)$, and the $j$ th phase is of length $O\left((\ln N)^{1 / 2^{j-1}}\right)$. All phases have the same basic structure, except for the last. Within each phase, there are $d$ subphases. In the $i$ th subphase, we assume that the $i$ th coordinate $z_{i, t}$ moves according to a biased random walk as previously described; in all other phases, we pessimistically assume that it moves according to an unbiased random walk. Our goal is to show that in each phase, each dimension moves closer to zero; in particular, after the $j$ th phase, all the $z_{i, t}$ are at $O\left((\ln N)^{1 / 2^{j}}\right)$ with constant probability. This is shown using Chernoff bounds (see Theorem 7 of Reference [15] for the corresponding calculation). It follows that after $O(\ln \ln \ln N)$ phases, each $z_{i, t}$ will be bounded by a constant with 
probability $\Omega(1)^{O(\ln \ln \ln N)}=\Omega\left(1 /(\ln \ln N)^{c}\right)$. At that point, there is a constant probability that the target vertex will be reached in a constant number of steps.

Putting this all together now yields the theorem that the cover time for the 2-cobra random walk on $[0, N]^{d}$ is $O(N)$ where the order notation hides constant factors that can depend on $d$. Lemma 3.4 shows that for each dimension $i, z_{i, t}$ hits 0 within the first $64 d^{2} N^{2}$ steps with high probability. Hence, by Lemma 3.5, after $64 d^{2} N^{2}$ steps all $z_{i, t}$ are at most $c_{d} \ln N$ with high probability. We are therefore in a state where we reach the target vertex within an additional $O(\ln N)$ steps with probability $\Omega\left(1 /(\ln \ln N)^{c}\right)$. If we have not reached the target vertex, however, we are still within $c_{d} \ln N$ distance in each dimension with high probability. That is, let $\mathcal{E}_{1}$ be the event that we did not reach the target vertex within the $\alpha \ln N$ steps of Lemma 3.6, and let $\mathcal{E}_{2}$ be the event that some $z_{i, t}$ is more than $c_{d} \ln n$ after those $\alpha \ln N$ steps. We have by Lemma 3.5 that this is a low probability event (at least up through an additional $36 d^{2} N^{2}$ steps), so we can consider a polylogarithmic number of repeated trials of $\alpha \ln N$ steps. As each trial succeeds with probability $\Omega\left(1 /(\ln \ln N)^{c}\right)$, we can conclude that we hit the target vertex with probability at least $1-O\left(1 / N^{d+1}\right)$ within $O(N)$ steps. It follows via a union bound that with probability $1-O(1 / N)$ all vertices are hit within $O(N)$ steps, from which it readily follows that the cover time is $O(N)$.

We remark that this proof, while achieving $O(N)$ bounds, appears quite loose in the constant factors. As our proof does not directly take advantage of the large number of pebbles within the system, we believe the bounds could be tightened with respect to the dependence on $d$. Even with this bound, we expect there remains further work to fully understand the behavior of cobra walks on grids.

We also remark that the multi-step case analysis used in Lemma 3.1 can similarly be used to show that 2-cobra walks on $k$-ary trees have cover times that are proportional to the graph's diameter when $k=2$ or $k=3$. We conjecture that in fact the cover time for 2-cobra walks on $k$-ary trees is proportional to the diameter for every constant $k$, where the constant of proportionality may depend on $k$, similar to Theorem 3.2 for grids.

\section{COVER TIME FOR A GRAPH WITH ARBITRARY CONDUCTANCE}

In this section, we significantly improve and extend the results first developed in Reference [15], which provided an $O\left(\log ^{2} n\right)$ bound for the cover time of a $k$-cobra walk on a $d$-regular expander with an expansion only achieved by graphs such as random $d$-regular expanders and Ramanujan expanders. Here, we provide the first known bound for the cover time of cobra walks on a $d$-regular graph of arbitrary conductance $\Phi$. While the upper bound is not useful for graphs with very low conductance, there are a wide class of graphs beyond expanders for which this guarantees rapid coverage, e.g., the hypercube, power-law graphs, and random geometric graphs. For this section and the rest of the article, we work exclusively with 2-cobra walks, and use cobra walks to mean 2-cobra walks where the meaning is clear.

Theorem 4.1. Let $G$ be a d-regular graph with conductance $\Phi_{G}$. Then a cobra walk starting at any vertex will cover $G$ in $O\left(d^{4} \Phi_{G}^{-2} \log ^{2}(n)\right)$ rounds, with high probability.

From this general bound, we have the following corollary, which improves the previous result of Reference [15].

COROLLARY 4.2. Let $G$ be a bounded-degreed-regular $\epsilon$-expander graph. Then a cobra walk starting at any vertex $v$ will cover $G$ in $O\left(\log ^{2}(n)\right)$ rounds with high probability when d is specifically bounded by a constant.

Due to the extreme difficulty of analyzing the progress of a cobra walk explicitly, we follow Reference [15] and analyze a process we call $W_{\text {alt }}$ that, while conceptually similar to a cobra walk, 
has more structured rules that allow us to analyze walks taken by individual pebbles with only limited dependence on one another. Furthermore, this process stochastically dominates the cobra walk when starting from the same vertex, with respect to the time to cover all of the vertices. Any upper bound on the cover time for this process therefore automatically applies to the cover time of a cobra walk as well. We define the process as follows.

Definition 4.3. $W_{\text {alt }}$. We start with some number of pebbles $z \geq 1$ distributed arbitrarily among the vertex set $V$, including the possibility for more than one pebble to start at the same vertex. Furthermore, we assume that the pebbles have a total ordering, and that each pebble knows its position in the ordering. In this process, unlike in the cobra walk, no pebbles split or coalesce-the total number of pebbles is an invariant. Pebbles interact with one another according to two simple rules. For each timestep:

(1) If one or two pebbles are co-located in time and space: at vertex $v$ at time $t$, each pebble chooses a random neighbor from $N(v)$ independently u.a.r. and moves to that vertex.

(2) If three or more pebbles are at $v$ at time $t$, the two pebbles with the lowest order each pick a vertex independently from $N(v)$ uniformly at random and move to their chosen vertex. Label these vertices $u, w$ (keeping in mind that $u=w$ is allowed). The remaining pebble(s) at $v$ then each independently pick $u$ with probability $1 / 2$ or $w$ with probability $1 / 2$ and move to the vertex they have chosen.

The process $W_{\text {alt }}$ can be viewed, at a single step, as a coalescing random walk in which the threshold for coalescence is three pebbles at the same vertex, rather than the standard two. As an added condition, the third and higher pebbles at a vertex (with respect to the total ordering of pebbles) chooses which of the first two pebbles to coalesce with via an unbiased coin flip.

If we are observing, for a single timestep, a vertex $v$ at which two or more pebbles (or zero, trivially) have landed, we would be unable to distinguish between a cobra walk and a $W_{\text {alt }}$ process. On the other hand, if we observe a vertex $v$ at which a single pebble has landed, we would be able to distinguish. In $W_{\text {alt }}$, in the next step, the pebble at $v$ will act like a simple random walk and move to a single neighbor. On the other hand, in the cobra walk, there is some probability $p$ two neighbors will receive a pebble from $v$, and probability $1-p$ only one neighbor will. Thus, the active set of $W_{\text {alt }}$ can be viewed as a (possibly proper) subset of the active set of a cobra walk when both are started from the same initial state. Therefore, at any future time $t$, the active set of a cobra walk stochastically dominates the active set of $W_{\text {alt }}$. This then yields the claim that the cover time of $W_{\text {alt }}$ stochastically dominates the cover time of the cobra walk.

Finally, for technical reasons, we make the $W_{\text {alt }}$ process a lazy process. That is, at each step, with probability $1 / 2$, all pebbles remain in their same position. With probability $1 / 2$, a step proceeds with the probabilities described above. (Thus, to obtain the unconditioned probabilities of any particular action, we need to multiply the above probabilities through by $1 / 2$.)

LEMmA 4.4. Let $G$ be a d-regular graph. Let $S \subset V$ be a subset of the vertices of $G$ such that $|S|<$ $n / 2$. Consider $C$, a cobra walk which begins at all the vertices of $S$, and $W, a W_{\text {alt }}$ process in which any number of pebbles are initially arbitrarily distributed among the vertices of $S$. Let $\tau_{C(S)}$ be the first time all the vertices are covered by $C$ and let $\tau_{W(S)}$ be the first time all the vertices are covered by $W$. Then for any time $T$, the probability that $\tau_{W(S)} \geq T$ is at least the probability that $\tau_{C(S)} \geq T$.

Proof. Our proof proceeds in two parts. In the first main part, we establish that the cover time of $C$ is stochastically dominated by the cover time of the non-lazy $W_{\text {alt }}$ process, which we refer to as $W^{\prime}$ in this proof. The second part establishes that the cover time of $W^{\prime}$ is stochastically dominated by the cover time of $W$, the lazy $W_{\text {alt }}$ process. 
For the first part, we use a standard coupling argument over Markov chains and partial ordered sets (e.g., see Reference [27]). For any step $t$, let random variables $A_{t}(C)\left(\right.$ resp., $\left.A_{t}\left(W^{\prime}\right)\right)$ denote the set of active vertices in $C$ (resp., $W^{\prime}$ ) after the end of step $t$. Each of the walks $C$ and $W^{\prime}$ defines a Markov chain over $A_{t}(C)$ and $A_{t}\left(W^{\prime}\right)$, respectively. Let $M(C)$ and $M\left(W^{\prime}\right)$, respectively, denote the transition matrices of the associated Markov chains. We establish that $M(C)$ stochastically dominates $M\left(W^{\prime}\right)$ by showing the stochastic domination among partially ordered sets given by one step of the Markov chain: for any sets $S_{t}$ and $S_{t}^{\prime}$ such that $S_{t}^{\prime} \subseteq S_{t}$, we show that the random variable $A_{t+1}(C) \mid A_{t}(C)=S_{t}$ stochastically dominates $A_{t+1}\left(W^{\prime}\right) \mid A_{t}\left(W^{\prime}\right)=S_{t}^{\prime}$.

To establish the stochastic dominance of $A_{t+1}(C) \mid A_{t}(C)=S_{t}$ over $A_{t+1}\left(W^{\prime}\right) \mid A_{t}\left(W^{\prime}\right)=S_{t}^{\prime}$, we couple the two probabilistic processes as follows. Consider time $t$, where $S_{t}$ and $S_{t}^{\prime}$ are the active sets in $C$ and $W^{\prime}$, respectively. In step $t+1$, we conduct two 1-hop random walks from each active vertex $v$ of the cobra walk. If $v$ has at least two pebbles, then we simulate the two 1-hop walks by the two 1-hop walks from the first two pebbles at $v$ in $W^{\prime}$. After this simulation, we find that the active vertices in $C$ at time $t+1$ include all of the vertices with pebbles in $W^{\prime}$ at time $t+1$. If $v$ has only one pebble, then the cobra walk process has additional randomness that could lead to new vertices becoming active. By a standard argument (e.g., see [27]), this establishes a coupling $\left(\widehat{A}_{t}(C), \widehat{A}_{t}\left(W^{\prime}\right)\right)$ of $A_{t}(C)$ and $A_{t}\left(W^{\prime}\right)$ such that $\widehat{A}_{t}(C)$ is a superset of $\widehat{A}_{t}\left(W^{\prime}\right)$. This coupling ensures that the cover time of $C$ is stochastically dominated by the cover time of $W$, thus completing the proof of the lemma.

The stochastic dominance of the cover time of $W$ over that of $W^{\prime}$ is straightforward. We establish this claim by coupling $W$ with the lazy version of $W^{\prime}$, which follows exactly the same transitions as $W^{\prime}$ except that it opts to stay where it currently is with probability $1 / 2$. For any time $s$, the sequence of active sets obtained in the lazy version of $W^{\prime}$ until time $s$ is a subset of the active sets of $W^{\prime}$ until time $s$. In this coupling, the set of vertices covered by $W$ is always a subset of the set of vertices covered by $W^{\prime}$, thus implying that the cover time of $W$ stochastically dominates the cover time of $W^{\prime}$.

We are now ready to prove Theorem 4.1. This proof uses the machinery developed in the proofs of Reference [15, Theorem 16 and Lemma 17]. We first make note of the new ideas needed to provide a general bound on the cover time in terms of the conductance of the graph. For completeness, we have refactored the proof with the changes necessary to prove our conductance claim and present it in its entirety here.

One significant difference with previous work is the following. The previous analysis consisted of two stages, with the first stage providing an exponential growth in the number of pebbles, but requiring the graph to have extremely high expansion. In contrast, we begin our analysis of $W_{\text {alt }}$ with a large number of pebbles, all of which are located at a single initial, arbitrary vertex $v$, and compare this to a cobra walk that starts at $v$. In the first stage of Reference [15], a cobra walk process was analyzed directly and it was shown that after $O(\log n)$ rounds, the size of the cobra walk went from one vertex in the active set to $\delta n$ vertices in the active set, with high probability. However, one restrictive requirement was that the (vertex) expansion $\epsilon$ of the graph $G$ needed to be extremely high, satisfying the inequality:

$$
\frac{1}{\epsilon^{2}(1-\delta)+\delta}>\frac{d\left(d e^{-k}+(k+1)\right)-\frac{k^{2}}{2}}{d\left(e^{-k}+(k-1)\right)-\frac{k^{2}}{2}},
$$

where $k$ is the branching factor of the cobra walk (2, for our purposes) and $d$ is the degree of $G$. Only some families of expanders, such as Ramanujan graphs and random regular graphs with high degree, would satisfy this condition. Once the cobra walk reaches at least $\delta n$ active vertices, we replace the cobra walk with a $W_{\text {alt }}$ in which we position one $W_{\text {alt }}$ pebble at each of $\delta n$ of the 
vertices that were active in the cobra walk at the time at which we perform the swap. The analysis then proceeds from this point to show that every vertex will have been visited by at least one pebble of $W_{\text {alt }}$ with high probability in $O\left(\log ^{2} n\right)$ time.

Since the stochastic dominance of the cover time of $W_{\text {alt }}$ over a cobra walk holds for all starting distributions of the pebbles on the vertices of $G$, it also therefore holds for the starting distribution in which all $\delta n$ pebbles begin at the same vertex. This allows us to work exclusively with a $W_{\text {alt }}$ in which all $\delta n$ pebbles begin at the same vertex for the entire analysis, bypassing the analysis of the cobra walk as it grows from one active node to a linear number of active nodes and therefore dropping the requirement that $G$ have extremely high expansion.

The second contribution of our new analysis is the derivation of bounds on the probability of coverage of vertices in terms of the conductance of the graph. As a consequence, we are able to derive bounds that hold for regular graphs with arbitrary values of $d$ and arbitrary $\Phi_{G}$.

Proof of Theorem 4.1. To prove the theorem, by Lemma 4.4, it suffices to consider a $W_{\text {alt }}$ with $\delta n$ pebbles beginning at the same vertex. We break up $W_{\text {alt }}$ into epochs of length $s$, where $s \in O\left(\Phi_{G}^{m} \log n\right)$, for $m$ a constant integer to be defined later. We show that each vertex $v$ has a constant probability of being hit by at least one pebble at the exact timestep the epoch ends. We then go through $O(\log n)$ epochs to boost the probability $v$ is covered to a sufficiently high probability and then take a union bound over every vertex to obtain the result.

We first prove that in a particular epoch vertex $v$ will be hit by at least one pebble with constant probability. Define $E_{i}$ to be the event that pebble $i$ for $i \in 1, \ldots,\lceil\delta n\rceil$ covers an arbitrary vertex $v$ at time $s$. Then the event that $v$ is hit by at least one pebble is $\bigcup E_{i}$. Note that for any two pebbles $i, j$ it is not safe to assume that $E_{i}$ and $E_{j}$ are independent, since $i$ and $j$ may cross paths during their walks and have their transition probabilities affected by the rules of $W_{\text {alt }}$. However, we can use a second-order inclusion-exclusion inequality to lower-bound the probability:

$$
\operatorname{Pr}\left[\bigcup_{i} E_{i}\right] \geq \sum_{i} \operatorname{Pr}\left[E_{i}\right]-1 / 2 \sum_{i, j: i \neq j} \operatorname{Pr}\left[E_{i} \cap E_{j}\right] .
$$

Thus, we need to show that for the righ-hand side, the first quantity is a constant and the second quantity is smaller.

The first part is fairly straightforward. $\operatorname{Pr}\left[E_{i}\right]$ can be viewed as the (marginal) probability that a simple random walk of pebble $i$ hits $v$ as time $s$. Indeed, if we only observe the movement of $i$ and ignore all other pebbles, its transition probability to any adjacent vertex from its current vertex is always $1 / d$, regardless of the number of pebbles at the current vertex. Since this reduces to analyzing a simple random walk, we can use the well-known result that after $O\left(\log n / \Phi_{G}^{2}\right)$ time the probability that $i$ will be at $v$ will be within a $\pm 1 / 2 n$ interval around $1 / n$, assuming that $G$ is regular and the stationary distribution is the normalized uniform vector. For example, we can use the following result from Reference [28] to bound the maximum difference between components of the probability distribution of the walk after $t$ steps and the stationary distribution:

$$
\left|p_{t}(v)-\pi(v)\right| \leq \sqrt{\frac{d(v)}{d(u)}} e^{-t v_{2}} \leq e^{-t \Phi_{G}^{2} / 2},
$$

where the quantity in the square root is 1 because of the regularity of the graph, and we have substituted $\Phi_{G}^{2} / 2$ for $v_{2}$, the second-largest eigenvalue of the normalized Laplacian of $G$. Thus, for $t>\frac{2 \log (2 n)}{\Phi_{G}^{2}}, \operatorname{Pr}\left[E_{i}\right] \geq 1 / 2 n$.

Next we establish an upper bound for $\operatorname{Pr}\left[E_{i} \cap E_{j}\right]$ using the joint (dependent) walks of pebbles $i$ and $j$. Without loss of generality, assume that $i$ has a lower order than $j$, and that if $i$ and $j$ are 
co-located in time and space, then not only is $i$ the lower order, but $j$ must behave like a third-orgreater priority pebble and choose the same vertex that $i$ next hops to with probability $1 / 2$. (Note that under this assumption, the total probability that $j$ moves to the same vertex as $i$ is $1 / 2+1 / 2 d$, a fact that will be important shortly).

We can view the random walks of $i$ and $j$ as a random walk over a graph with the topology of the tensor product graph $G \times G$. The tensor product graph has the Cartesian product $V(G) \times V(G)$ as its vertex set, and an edge set defined as follows: vertex $\left(u, u^{\prime}\right) \in V(G \times G)$ has an edge to $\left(v, v^{\prime}\right) \in$ $V(G \times G)$ if and only if $(u, v),\left(u^{\prime}, v^{\prime}\right) \in E(G)$. The random walk we construct on $G \times G$ is slightly different than a simple random walk on the same graph: we make the edges directed, and we attach weights to them such that the walk on the directed graph $D(G \times G)$ is isomorphic to the movement of pebbles $i, j$ in a $W_{\text {alt }}$ on $G$.

We will show in the next lemma that the walk on $D(G \times G)$ has a stationary distribution, that this stationary distribution is close to $1 / n^{2}$, and that the walk converges rapidly to it. Thus, after $s$ steps, the probability that $i$ and $j$ are at the same vertex in $G$ is bounded from above by $2 /\left(n^{2}+n\right)+1 / n^{4}$.

Once we have established bounds for $\operatorname{Pr}\left[E_{i}\right], \operatorname{Pr}\left[E_{i} \cap E_{j}\right]$, we can apply them to the full expression and get

$$
\begin{aligned}
\operatorname{Pr}\left[\bigcup E_{i}\right] & \geq \sum_{i} \operatorname{Pr}\left[E_{i}\right]-\frac{1}{2} \sum_{i \neq j} \operatorname{Pr}\left[E_{j} \cap E_{i}\right] \\
& \geq \delta n \frac{1}{2 n}-\frac{1}{2}\left(\begin{array}{c}
\delta n \\
2
\end{array}\right)\left(\frac{2}{n^{2}+n}+\frac{1}{n^{4}}\right) \\
& \geq \frac{\delta}{2}-\frac{\delta}{4}\left(\delta n^{2}-n\right)\left(\frac{2}{n^{2}+n}+\frac{1}{n^{4}}\right) \\
& \geq \frac{\delta}{2}-\frac{2 \delta^{2}}{4} .
\end{aligned}
$$

Finally, to complete the proof, we have the following lemma:

Lemma 4.5. Let $G$ and s be defined as in Theorem 4.1. Let $i, j$ be two pebbles walking on $G$ according to the rules of $W_{\text {alt }}$. If $E_{i} \cap E_{j}$ are defined as the event in which $i$ and $j$ are both located at (arbitrarily chosen) vertex $v$ at time s, then $\operatorname{Pr}\left[E_{i} \cap E_{j}\right] \leq \frac{2}{n^{2}+2}+\frac{1}{n^{4}}$.

Proof. We first make a few observations about the topology of graph $G \times G$. There are two classes of vertices: the first class involves all vertices that have the form $(u, u)$ (and recall that $u \in V(G))$. A pebble at $(u, u)$ would correspond to two pebbles occupying $u$ in the paired walk on $i, j$ on $G$. Label the set of such vertices $S_{1}$. The cardinality of $S_{i}$ is $n$. The remaining vertices, which belong to what we label $S_{2}$, are of the form $(u, v)$, for $u \neq v$. There are $n^{2}-n$ such vertices. Also note that in the undirected graph $G \times G$, each vertex has degree $d^{2}$. Further, every vertex in $S_{1}$ will have $d$ neighbors also in $S_{1}$, by virtue of the regularity of $G$.

Many of the spectral properties of $G$ apply to $G \times G$. Because $G$ is an $\epsilon$-expander, the transition probability matrix of a simple random walk on $G$ has a second eigenvalue $\alpha_{2}(G)$ bounded away from zero by a constant. It is well known (c.f. Reference [23]) that the transition probability matrix of a simple random walk on $G \times G$ will have $\alpha_{2}(G \times G)=\alpha_{2}(G)$, which we will refer to as $\alpha_{2}$ henceforth.

Next, we transform the (undirected) graph $G \times G$ into a directed graph $D(G \times G)$ according to the following rules: For every undirected edge $(x, y)$ in $G \times G$, we replace it with two directed edges: $x \rightarrow y$ and $y \rightarrow x$. As mentioned, every vertex in $S_{1}$ will have $d$ neighbors also in $S_{1}$, meaning that there will be one directed $\operatorname{arc} x \rightarrow y$ for every vertex $x \in S_{1}$ and $y \in N(x)$ for $y$ in either $S_{1}, S_{2}$. We next add an additional $d$ copies of edge $x \rightarrow y$ for every such original edge. It is relevant 
for the analysis to note that the regularity of the subgraph of $G \times G$ induced on $S_{1}$ implies that every vertex in $\mathcal{D}(G \times G)$ will have an equal number of out and in arcs. Therefore $\mathcal{D}(G \times G)$ is an Eulerian digraph, a fact that will be important when calculating the stationary distribution of a walk on this graph.

Next, we calculate the transition probabilities of a random walk on $\mathcal{D}(G \times G)$. An outgoing edge from $x$ is picked with probability $1 / d$. However, when we compute the probability of transitioning to a neighboring vertex, there is a difference between nodes in $S_{1}$ and $S_{2}$. In $S_{2}$, each transition occurs with probability $1 / d^{2}$, as in the case of a symmetric walk. Note that this includes transitions to nodes in $S_{1}$ as well as within $S_{2}$. On the other hand, the probabilities of transitions from nodes in $S_{1}$ are modified. The probability of transitioning from a vertex $x \in S_{1}$ to one of its $d^{2}-d$ neighbors in $S_{2}$ is $1 / 2 d^{2}$. The probability of transition to another neighbor in $S_{1}$ is $(d+1) / 2 d^{2}$ (on account of the multiple edges). Thus, the walk on digraph $\mathcal{D}(G \times G)$ corresponds is isomorphic to the joint walk of pebbles $i, j$ on $G$ according to the rules of $W_{\text {alt }}$.

Because the walk on $D(G \times G)$ is irreducible, it has a stationary distribution $\pi$, which is the (normalized) eigenvector of the dominant eigenvalue of the transition matrix $M$ of the walk on $D(G \times G)$. Furthermore, because $D(G \times G)$ is Eulerian, the stationary distribution of vertex $x$ is exactly given by: $d^{+}(x) /|E|$, where $d^{+}(x)$ is the out-degree of $x$. Therefore, there are only two distinct values of the components of the stationary vector: for all $x \in S_{1}, \pi(x)=2 /\left(n^{2}+n\right)$, while for all $y \in S_{2}, \pi(y)=1 /\left(n^{2}+n\right)$.

A note of caution: Because $G$ and $G \times G$ have such nice spectral properties, and because $\mathcal{D}(G \times G)$ represents such a minor modification of $G \times G$ it would be natural to infer that $\mathcal{D}(G \times G)$ also has many of the same nice properties (particularly, the property of rapid convergence). However, it is often the case that properties of Markov chains on undirected graphs do not also hold for Markov chains on directed graphs. Thus, we must carefully check our rapid convergence hypothesis. Fortunately, following closely the work of Reference [10], we can indeed verify that the walk on $\mathcal{D}(G \times G)$ converges rapidly to its stationary distribution.

For succinctness of notation, denote $\mathcal{D}=D(G \times G)$. Consider the function $F_{\pi}: E(\mathcal{D}) \rightarrow \mathfrak{R}$ given by $F_{\pi}(x, y)=\pi(x) P(x, y)$, where $\pi(x)$ is the $x$ th component of the stationary distribution of the walk on $\mathcal{D}$ and $P(x, y)$ is the associated transition probability moving from $x$ to $y$. Then $F_{\pi}$ is the circulation associated with the stationary vector as shown in Lemma 3.1 of Reference [10]. Note that a circulation is any such function that satisfies a balance equation: $\sum_{u, u \rightarrow v} F(u, v)=\sum_{w, v \rightarrow w} F(v, w)$.

There is a Cheeger constant for every directed graph, defined as

$$
h(G)=\inf _{S} \frac{F_{\pi}(\partial S)}{\min \left\{F_{\pi}(S), F_{\pi}(\bar{S})\right\}},
$$

where $F_{\pi}(\partial S)=\sum_{u \in S, v \notin S} F(u, v), F(v)=\sum_{u, u \rightarrow v} F(u, v)$, and $F(S)=\sum_{v \in S} F(v)$ for a set S. Furthermore, Theorem 5.1 of Reference [10] shows that the second eigenvalue, $\lambda$, of the Laplacian of $\mathcal{D}$ satisfies

$$
2 h(\mathcal{D}) \geq \lambda \geq \frac{h^{2}(\mathcal{D})}{2} .
$$

The Laplacian of a directed graph is defined somewhat differently than the Laplacian of an undirected graph. However, because we will not use the Laplacian directly in our analysis, we refer the reader to Reference [10] for the definition. We will directly bound the Cheeger constant for $\mathcal{D}$, and hence produce a bound on the second eigenvalue of the Laplacian. This second bound will then be used to provide a bound on the convergence of the chain to its stationary distribution. 
First, without loss of generality, assume that $F_{\pi}(S)$ is smaller than its complement. Furthermore, assume that $S$ is the set that satisfies the inf condition in the Cheeger constant. We have $F_{\pi}(\partial S)=$ $\sum_{x \rightarrow y, x \in S, y \in \bar{S}} \pi(x) P(x, y)$, and $F_{\pi}=\sum_{x \rightarrow y, x \in S} \pi(x) P(x, y)$. The first sum occurs over all (directed) edges that cross the cut of $S$, while the second sum occurs over all edges leaving vertices in $S$ : the numerator and denominator of the conductance $\Phi_{G \times G}$. Thus we can provide a lower bound for the entire Cheeger constant:

$$
\begin{aligned}
h(\mathcal{D}) & =\inf _{S} \frac{F_{\pi}(\partial S)}{\min \left\{F_{\pi}(S), F_{\pi}(\bar{S})\right\}} \\
& \geq \Phi_{G \times G} \frac{P_{\min } \pi_{\min }}{P_{\max } \pi_{\max }} \\
& =\Phi_{G \times G} \cdot \frac{\frac{1}{4 d^{2}} \frac{1}{\left(n^{2}+n\right)}}{\frac{1}{2} \frac{2}{\left(n^{2}+n\right)}}=\frac{\Phi_{G \times G}}{4 d^{2}},
\end{aligned}
$$

where $P_{\max }$ is $1 / 2$ because of the lazy property of $W_{\text {alt }}$.

Next, we show that $G$ and $G \times G$ have the same conductance. As we noted earlier, $G$ and $G \times G$ have the same second-smallest Laplacian eigenvalue $v_{1}$. From Reference [30], we know that they have the same spectral gap $\gamma$, defined as $1-\lambda(G)$ where $\lambda$ is the second largest eigenvalue of the adjacency matrix of $G$. From Theorem 4.14, we also know that the spectral expansion (gap) of $G$ is equal to $\alpha \epsilon^{2} / 2$, where $\alpha$ is the fraction of edges that are self loops and $\epsilon$ is the edge expansion of $G$, which for $d$-regular graphs is the same as the conductance $\Phi_{G}$. Thus, $\Phi_{G}=\Phi_{G \times G}$.

We then apply the lower (directed) Cheeger inequality to have $\lambda \geq \frac{\Phi_{G}^{2}}{32 d^{4}}$.

We now show a bound on convergence of the walk on $\mathcal{D}$ to the stationary distribution. To measure distance from the stationary distribution, we use the $\Xi$-square distance:

$$
\Delta^{\prime}(t)=\max _{y \in V(\mathcal{D})}\left(\sum_{x \in V(\mathcal{D})} \frac{\left(P^{t}(y, x)-\pi(x)\right)^{2}}{\pi(x)}\right)^{2} .
$$

It is straightforward to show that any distance in the $\Delta^{\prime}$ metric is no smaller than a distance using a total-variational distance metric. Hence the distribution of a random walk starting anywhere in $\mathcal{D}$ will be close to its stationary distribution w.r.t. each component vertex.

We next apply Theorem 7.3 from Reference [10].

THEOREM 4.6. Suppose a strongly connected directed graph $G$ on $n$ vertices has Laplacian eigenvalues $0=\lambda_{0} \leq \lambda_{1} \leq \cdots \leq \lambda_{n-1}$. Then $G$ has a lazy random walk with the rate of convergence of order $2 \lambda_{1}^{-1}\left(-\log \min _{x} \pi(x)\right)$. Namely, after at most $t \geq 2 \lambda_{1}^{-1}\left(\left(-\log \min _{x} \pi(x)+2 c\right)\right.$ steps, we have

$$
\Delta^{\prime}(t) \leq e^{-c} \text {. }
$$

Recall that we provided a lower bound for $\lambda$, the second-smallest eigenvalue of the Laplacian of $\mathcal{D}$ in terms of the conductance of $\Phi_{G}$. We can thus apply the above theorem to show that after at most

$$
s=\frac{32 d^{4}}{\Phi_{G}^{2}}\left(\log \left(n^{2}+n\right)+4 \log \left(n^{2}\right)\right)
$$

steps we will have $\Delta^{\prime}(t) \leq \frac{1}{n^{4}}$. For the random walk on $\mathcal{D}$, after $s$ steps, a walk that starts from any initial distribution on $V(\mathcal{D})$ will be within $n^{-4}$ distance of the stationary distribution of any vertex. 
Mapping our analysis back directly to the coupled walk of $i, j$ on $G$, it then holds that $\operatorname{Pr}\left[E_{i} \cap\right.$ $\left.E_{j}\right] \leq 2 /\left(n^{2}+n\right)+1 / n^{4}$ when pebbles $i$ and $j$ start from any initial position.

It follows immediately that when $d$ is a constant and $G$ is any expander with conductance $\Phi_{G}$ bounded away from zero by a constant, a cobra walk will cover $G$ at most $O\left(\log ^{2} n\right)$ time.

\section{COVER TIME FOR GENERAL GRAPHS}

In this section, we show that the cover time of 2-cobra walks for general graphs is $O\left(m n^{3 / 4} \log n\right)$. For $\delta$-regular graphs, we obtain an improved bound of $O\left(n^{2-1 / \delta} \log n\right)$. We establish the bounds on the cover time by proving that for any start vertex $u$ and any target vertex $v$, the hitting time from $u$ to $v$, denoted by $H(u, v)$, is $O\left(m n^{3 / 4}\right)$ for general graphs and $O\left(n^{2-1 / \delta}\right)$ for $\delta$-regular graphs. We then apply Matthews' Theorem (Theorem 2.1), which actually gives a high probability result.

To analyze the cobra walk, we consider biased versions of the standard random walk; in each step, with some probability, instead of moving to a neighbor chosen uniformly at random, the walk moves to a vertex as determined by a memoryless controller whose aim is to reach a certain target vertex. The biased walk we analyze is a variant of $\epsilon$-biased walks defined in Reference [5]. In Section 5.1, we review the $\epsilon$-biased walks of Reference [5] and introduce and analyze our variant, which we call inverse-degree-biased walks. In Section 5.2 , we use $1 / \delta$-biased walks to derive an $O\left(n^{2-1 / \delta}\right)$ bound on the hitting time of the cobra walk on $\delta$-regular graphs, improving on the quadratic bound for standard random walks. In Section 5.3, we use inverse-degree-biased walks to derive an $O\left(\mathrm{mn}^{3 / 4}\right)$ bound on the hitting time of the cobra walk on nonregular graphs, improving on the cubic bound for standard random walks.

\subsection{Biased Random Walks}

An $\epsilon$-biased walk on $G$ is defined as follows: in each step, with probability $1-\epsilon$, one of the neighbors is selected uniformly at random, and the walk moves there; with probability $\epsilon$, a controller gets to select which neighbor to move to. The controller can be probabilistic, but it is time independent. The primary motivation for biased random walks is to study how much can a controller increase the occupancy probabilities at a targeted subset $S$ of the vertices in the stationary distribution. A central result of Reference [5] is the following lower bound achieved by an optimal controller in an $\epsilon$-biased walk.

Theorem 5.1 (Theorem 2 of Reference [5]). Let $G=(V, E)$ be a connected graph, $S \subset V$, $v \in S$ and $x \in V$. Let $\Delta(x, v)$ be the length of the shortest path between vertices $x$ and $v$ in $G$ and $\Delta(x, S)=$ $\min _{v \in S} \Delta(x, v)$. Let $\beta=1-\epsilon$. There is an $\epsilon$-bias strategy for which the stationary probability at $S$ (i.e., the sum of the stationary probabilities of $v \in S$ ) is at least

$$
\frac{\sum_{v \in S} d(v)}{\sum_{v \in S} d(v)+\sum_{x \notin S} \beta^{\Delta(x, S)-1} d(x)} .
$$

The bias in an $\epsilon$-biased walk is constant $(\epsilon)$ at each vertex. To analyze the cobra walk, we define a biased walk in which the bias available to the controller at each vertex of the graph varies with the vertex. Specifically, we define the inverse-degree-biased walk with target $x$ : if the walk is at $x$, then the next vertex visited is a neighbor of $x$ chosen uniformly at random; if the walk is at vertex $v \neq x$, then with probability $1-1 / d(v)$, one of the neighbors is selected uniformly at random and the walk moves there, and with probability $1 / d(v)$ a controller gets to select which neighbor to move to. The goal of the controller is to minimize the hitting time to $x$.

Our main motivation for introducing inverse-degree-biased walks is the following dominance argument. For any pair of vertices $u$ and $v$, let $H^{*}(u, v)$ denote the smallest hitting time to $v$ achievable by an inverse-degree-biased walk with target $v$, starting at $u$. Recall that $H(u, v)$ denotes the hitting time at $v$ for a cobra walk starting at $u$. 
LEMMA 5.2. For any pair of vertices $u$ and $v, H(u, v) \leq H^{*}(u, v)$.

Proof. Our proof is by a coupling argument. Consider the cobra walk starting at $u$ at time 0 . For any vertex $x$ and any time $t$, let $E_{t}^{*}(x)$ denote the event that $x$ is active in the cobra walk at the start of round $t$. For any vertex $x$, any neighbor $y$ of $x$, and any time $t$, let $P_{t}^{*}(x, y)$ be the probability that $y$ is active at the start of round $t+1$ conditioned on the event that $x$ is active at the start of round $t$. That is,

$$
P_{t}^{*}(x, y)=\operatorname{Pr}\left[E_{t+1}^{*}(y) \mid E_{t}^{*}(x)\right] .
$$

We next consider any inverse-degree-biased walk starting from $u$. Let $E_{t}(x)$ denote the event that the walk is at vertex $x$ at the start of round $t$. Let $P_{t}^{\prime}(x, y)$ be the probability that the inverse-degreebiased walk is at $y$ at the start of round $t+1$, conditioned on the event that the walk is at $x$ at the start of round $t$. By the definition of the cobra walk and the inverse-degree-biased walk, we have the straightforward derivation.

$$
\begin{aligned}
P_{t}^{*}(x, y) & =1-\left(1-\frac{1}{d(u)}\right)^{2} \\
& \geq \frac{2}{d(u)}-\frac{1}{d(u)^{2}} \\
& =\frac{1}{d(u)}+\left(1-\frac{1}{d(u)}\right) \frac{1}{d(u)} \\
& \geq P_{t}^{\prime}(x, y) .
\end{aligned}
$$

We now invoke a standard coupling argument to obtain that for any round $t$ and any vertex $x$, the probability that $x$ is active in the cobra walk at the start of round $t$ is at least the probability that the inverse-degree-biased walk is at $x$ at the start of round $t$. This also implies that the expected time for $u$ to become active in the cobra walk is at most the expected time to hit $x$ in the inversedegree-biased walk. Setting $x=v$ and choosing the inverse-degree-biased walk to be one that minimizes the hitting time to $v$ yields $H(u, v) \leq H^{*}(u, v)$.

\section{2 $O\left(n^{2-1 / \delta}\right)$ Hitting Time for $\delta$-regular Graphs}

For the case of $\delta$-regular graphs, the inverse-degree-biased walk with target $v$ is exactly a $1 / \delta$ biased walk with target $v$. We invoke Theorem 5.1 for $\delta$-regular graphs with $\beta=1-1 / \delta$, and letting $S$ equal $\{v\}$. We calculate a lower bound on stationary probability at $v$ of the $1 / \delta$-biased walk with target $v$, we need to determine the maximum value that $\sum_{x \neq v} \beta^{\Delta(x, v)-1}$ can take. We calculate this maximum value, over all graphs with maximum degree $\delta$, hence yielding an upper bound for $\delta$-regular graphs as well. Let $n_{i}$ denote the number of vertices within $i$ hops of $v$. Then, we have

$$
\sum_{x \neq v} \beta^{\Delta(v, x)-1}=\sum_{i \geq 1} n_{i} \beta^{i}
$$

Since the degree is at most $\delta$, we have the following constraints on the $n_{i}$ s, given by the number of vertices within a certain number of hops from $v$ :

$$
\sum_{1 \leq i \leq j} n_{i} \leq \min \left\{n-1, \delta\left(1+\sum_{1 \leq i<j}(\delta-1)^{i}\right)\right\} .
$$


Since $\beta<1$, and the total number of vertices is bounded by $n$, the sum $\sum_{i \geq 1} n_{i} \beta^{i}$ is maximized when

$$
\begin{aligned}
& n_{1}=\delta \\
& n_{j}=\delta(\delta-1)^{j} 1<j<L,
\end{aligned}
$$

where $L$ is the smallest $j$ such that $\delta\left(1+\sum_{1 \leq i<j}(\delta-1)^{i}\right)$ is at least $n-1$. Then, we have $n_{L}=$ $n-1-\sum_{j<L} n_{j}$. With elementary algebra, we calculate the value of $L$ as

$$
L=\log _{(\delta-1)}\left[\frac{(n-1)}{\delta}(\delta-2)+1\right] .
$$

We now bound $\sum_{x \neq v} \beta^{\Delta(x, v)-1}$ as follows:

$$
\begin{aligned}
\sum_{x \neq v} \beta^{\Delta(v, x)-1}= & \delta \beta^{0}+(\delta-1) \delta \beta^{2}+\delta(\delta-1)^{2} \beta^{2} \\
& +\cdots+\delta(\delta-1)^{L-1} \beta^{L-1} \\
= & d\left(\sum_{i=0}^{L-1}((\delta-1) \beta)^{i}\right) \\
= & d\left[\frac{((\delta-1) \beta)^{L}-1}{(\delta-1) \beta-1}\right] \\
\leq & C\left[((\delta-1) \beta)^{L}\right],
\end{aligned}
$$

where $C=\delta /((\delta-1) \beta-1)$.

With some minimal algebra, we can see that

$$
(\delta-1)^{\log _{\delta-1}(n-1)(\delta-2) / \delta+1}=(n-1)(\delta-2) / \delta+1<n
$$

and that

$$
\begin{aligned}
\beta^{L} & =(1-1 / \delta)^{\log _{\delta-1}(n-1)(\delta-2) /(\delta-1)+1} \\
& \leq(\delta-1)^{(-1 / \delta) \log _{\delta-1}((n-1)(\delta-1) /(\delta-2)+1)} \\
& =\left[\frac{(n-1)(\delta-2)+\delta}{\delta}\right]^{-1 / \delta}<n^{-1 / \delta}
\end{aligned}
$$

Therefore, the stationary distribution at $v$ is at least $1 /\left(1+n^{1-1 / \delta}\right)$, giving a return time to $v$ of at most $1+n^{1-1 / \delta}$. Using the tree-traversal argument and noting that it will take, in expectation, $d$ returns to current vertex $v$ to make one "progress" step in the traversal in expectation for a total cost of $\delta n^{1-1 / \delta}$ per progress step. Thus, the total cost of coverage will be $2 \delta C n^{2-1 / \delta}=O\left(n^{2-1 / \delta}\right)$ for constant $\delta$.

TheOREM 5.3. Let $G$ be a finite, $\delta$-regular graph. Then the hitting time of a cobra walk on $G$ is in $O\left(n^{2-\frac{1}{\delta}}\right)$, for any constant $\delta$.

\section{3 $O\left(m n^{3 / 4}\right)$ Hitting Time for General Graphs}

To bound $H(u, v)$ in a general graphs, let $P$ be a shortest-hop path from $u$ to $v$, given by the sequence of vertices $u=u_{0}, u_{1}, u_{2}, \ldots, u_{|P|}=v$. Then

$$
H(u, v) \leq \sum_{i=0}^{|P|-1} H\left(u_{i}, u_{i+1}\right) .
$$


Let $R(x)$ denote the minimum return time to $x$ among all inverse-degree-biased walks with target $x$. We have

$$
R(x)=1+\sum_{y \in N(x)} \frac{H^{*}(y, x)}{d(x)} .
$$

Furthermore, we have

$$
\begin{aligned}
H\left(u_{i}, u_{i+1}\right) & \leq 1+\sum_{y \in N\left(u_{i}\right), y \neq u_{i+1}} H\left(y, u_{i+1}\right) / d\left(u_{i}\right) \\
& \leq 1+\sum_{y \in N\left(u_{i}\right), y \neq u_{i+1}} \frac{H\left(y, u_{i}\right)+H\left(u_{i}, u_{i+1}\right)}{d\left(u_{i}\right)} \\
& \leq 1+\sum_{y \in N\left(u_{i}\right), y \neq u_{i+1}} \frac{H^{*}\left(y, u_{i}\right)+H\left(u_{i}, u_{i+1}\right)}{d\left(u_{i}\right)} \\
& \leq R\left(u_{i}\right)+\sum_{y \in N\left(u_{i}\right), y \neq u_{i+1}} H\left(u_{i}, u_{i+1}\right) / d\left(u_{i}\right) \\
& =R\left(u_{i}\right)+\left(1-\frac{1}{d\left(u_{i}\right)}\right) H\left(u_{i}, u_{i+1}\right) .
\end{aligned}
$$

(The second step follows from the fact that the hitting time from $y$ to $u_{i+1}$ is at most the sum of the hitting times from $y$ to $u_{i}$ and from $u_{i}$ to $u_{i+1}$. The third step follows from Lemma 5.2. The fourth step follows from Equation (6). The final equality holds since $u_{i}$ has $d\left(u_{i}\right)$ neighbors.)

The above equation easily yields $H\left(u_{i}, u_{i+1}\right) \leq d\left(u_{i}\right) R\left(u_{i}\right)$, which combined with Equation (5) yields

$$
H(u, v) \leq \sum_{i=0}^{|P|-1} H\left(u_{i}, u_{i+1}\right) \leq \sum_{i=0}^{|P|-1} d\left(u_{i}\right) R\left(u_{i}\right) .
$$

We prove the following extension of Theorem 5.1, applied to inverse-degree-biased walks. For the sake of completeness, we include the proof, which essentially mimics the proof of Theorem 2 in Reference [5].

LEMmA 5.4. Let $G=(V, E)$ be a connected graph and $S$ be an arbitrary subset of $V$. For a given path $P$, let $\sigma(P)$ denote the product $\prod_{y \in P}(1-1 / d(y))$. For $v \in S$ and $x \in V-S$, let $\widehat{\sigma}(x, v)$ denote the maximum, over all paths $P$ from $x$ to $v$, of $\sigma(P)$. Let $\widehat{\sigma}(x, S)$ denote the minimum, over all $v \in S$, of $\widehat{\sigma}(x, v)$. There is an inverse-degree-biased walk for which the stationary probability at $S$ (i.e., the sum of the stationary probabilities of $v \in S$ ) is at least

$$
\frac{\sum_{v \in S} d(v)}{\sum_{v \in S} d(v)+\sum_{x \notin S}(\widehat{\sigma}(x, S) d(x))} .
$$

Proof. Define a probability distribution $\pi^{M}: \pi^{M}(v)=\gamma d(v)$ for $v \in S$ and $\pi^{M}(x)=$ $\gamma \widehat{\sigma}(x, S) d(x)$ for $x \notin S$, where

$$
\gamma=\frac{1}{\sum_{v \in S} d(v)+\sum_{x \notin S}(\widehat{\sigma}(x, S) d(x))} .
$$

Let $M$ be the transition probability matrix for which the stationary distribution given by Metropolis Theorem [5, Theorem 1] [25] is $\pi^{M}$. Define the following transition probability matrix: $P_{x, x}=0$ for all $x \in V$, and for $y \neq x, P_{x, y}=M_{x, y} /\left(1-M_{x, x}\right)$. We now argue that $M_{x, y} \geq(1-1 / d(x)) / d(x)$ 
for any neighbor $y$ of $x$. By the Metropolis Theorem, $M_{x, y}$ is either $1 / d(x)$ or $\pi^{M}(y) /\left(d(y) \pi^{M}(x)\right)$. In the former case, the desired lower bound on $M_{x, y}$ is trivial. In the latter case, we have

$$
M_{x, y}=\frac{\widehat{\sigma}(y, S)}{d(x) \widehat{\sigma}(x, S)} \geq \frac{1-1 / d(x)}{d(x)},
$$

where the last inequality follows from the fact that $\widehat{\sigma}(y, S) \geq(1-1 / d(x)) \widehat{\sigma}(x, S)$. We thus obtain $P_{x, y} \geq M_{x, y} \geq(1-1 / d(x)) / d(x)$ for any neighbor $y$ of $x$. So $P$ is a transition probability matrix of an inverse-degree-biased walk.

We next show that for each vertex in $S$, its stationary probability under $P$ is at least its stationary probability under $M$. Let $H^{M}(x, v)$ and $H^{P}(x, v)$ denote the hitting time to $v$ under the walks defined by $P$ and $M$, respectively, with start vertex $x$. For each vertex $x \neq v$, we have

$$
H^{M}(x, v)=1+M_{x, x} H^{M}(x, v)+\sum_{y \in N(x)} M_{x, y} H^{M}(y, v),
$$

which yields

$$
\begin{aligned}
H^{M}(x, v) & =\frac{1}{1-M_{x, x}}+\sum_{y \in N(x)} \frac{M_{x, y}}{1-M_{x, x}} H^{M}(y, v) \\
& \geq 1+\sum_{y \in N(x)} P_{x, y} H^{M}(y, v) .
\end{aligned}
$$

By Reference [5, Lemma 1], for every $x \in V$ and $v \in S, H^{M}(x, v) \geq H^{P}(x, v)$. If we denote the return time to vertex $v$ in the walks under $P$ and $M$ by $R^{P}(v)$ and $R^{M}(v)$, respectively, we obtain that

$$
\begin{aligned}
R^{M}(v) & =1+\sum_{y \in N(v)} M_{v, y} H^{M}(y, v) \\
& =1+\sum_{y \in N(v)} P_{v, y} H^{M}(y, v) \\
& \geq 1+\sum_{y \in N(v)} P_{v, y} H^{P}(y, v) \\
& =R^{P}(v)
\end{aligned}
$$

We thus have

$$
\pi^{P}(S) \geq \pi^{M}(S)=\frac{\sum_{v \in S} d(v)}{\sum_{v \in S} d(v)+\sum_{x \notin S} d(x) \widehat{\sigma}(x, S)} .
$$

COROLlary 5.5. Let $v$ be an arbitrary vertex of $G$. There exists a bias strategy for the inversedegree-biased walk such that the return time to $v$ in the walk is at most

$$
\frac{d(v)+\sum_{x \neq v} \widehat{\sigma}(x, v) d(x)}{d(v)} .
$$

Proof. The result follows from Lemma 5.4, setting $S=\{v\}$ and noting that the return time to $v$ equals the inverse of the stationary probability at $v$.

For our analysis, it is more convenient to work with an upper bound on $\widehat{\sigma}(x, v)$ (for each $x$ and $v)$ as given in the following lemma. Let $p(y, x)$ denote the weight of the shortest path from $y$ to $x$ in $G$, where we assign a weight of $1 / d(z)$ for each vertex $z$.

LEMMA 5.6. For all vertices $x$ and $v, \widehat{\sigma}(x, v)$ is at most $e^{-p(x, v)}$. 
Proof. Let $P^{\prime}$ denote a path between $x$ and $v$ such that $\widehat{\sigma}(x, v)=\sigma(P)$. Then, we have

$$
\widehat{\sigma}(x, v)=\prod_{y \in P^{\prime}}(1-1 / d(y)) \leq \prod_{y \in P^{\prime}} e^{-1 / d(y)}=e^{-p(x, v)} .
$$

LEMma 5.7. For start vertex $u$ and target $v$, let $P$ be a shortest-hop path from $u$ to $v$, given by the sequence of vertices $u=u_{0}, u_{1}, u_{2}, \ldots, u_{|P|}=v$. Then, we have

$$
\sum_{i=0}^{|P|-1} d\left(u_{i}\right) R\left(u_{i}\right)=O\left(m n^{3 / 4}\right) .
$$

Proof. By Lemma 5.2 and Corollary 5.5, we have

$$
\begin{aligned}
\sum_{i=0}^{|P|-1} d\left(u_{i}\right) R\left(u_{i}\right) & \leq \sum_{i=0}^{|P|-1} d\left(u_{i}\right) R\left(u_{i}\right) \\
& \leq \sum_{i=0}^{|P|-1} d\left(u_{i}\right)\left(\frac{d\left(u_{i}\right)+\sum_{x \neq u} \widehat{\sigma}(x, u) d(x)}{d\left(u_{i}\right)}\right) \\
& \leq \sum_{i=0}^{|P|-1}\left(d\left(u_{i}\right)+\sum_{x \neq u_{i}} d(x) e^{-p\left(x, u_{i}\right)}\right) \\
& \leq 3 n+\sum_{i=1}^{|P|-1} \sum_{x \neq u_{i}} d(x) e^{-p\left(x, u_{i}\right)} \\
& \leq 3 n+\sum_{x \in V}\left(d(x) \sum_{i=1}^{|P|-1} e^{-p\left(x, u_{i}\right)}\right) .
\end{aligned}
$$

(The second step follows from Corollary 5.5. The third step follows from Lemma 5.6. The fourth step follows from the elementary claim that the sum of the degrees of vertices along any shortest path in an $n$-vertex graph is at most $3 n$; e.g., see Reference [19, Proof of Theorem 2.1]. The final step is obtained by rearranging the summations.)

We next place an upper bound on $\sum_{x \in V}\left(d(x) \sum_{i=1}^{|P|-1} e^{-p\left(x, u_{i}\right)}\right)$. We claim that the number of vertices $u_{i}$ in $P$ to which any vertex $x$ has paths of length at most $L$ is at most $2 L$. This is because two vertices $a$ and $b$ that are more than $2 L$ hops away in $P$ would have a path of $2 L$ hops via $x$, a contradiction.

Consider a vertex $u_{i}$, for some $i$. Consider any path from $x$ to $u_{i}$ that minimizes $p\left(x, u_{i}\right)$. No vertex in the graph has edges to more than $\sqrt{n}$ vertices along this path. If it did, then we could short-cut and decrease the sum of the reciprocals of the degrees $1 / \sqrt{n}<\sqrt{n} /(n-1)$. Therefore, any path that minimizes $p\left(x, u_{i}\right)$ has a total degree of at most $n^{3 / 2}$. If the length $L$ of the path is less than $\sqrt{n}$, then $p\left(x, u_{i}\right) \geq 0$; otherwise, $p\left(x, u_{i}\right) \geq L^{2} / n^{3 / 2}$.

We now show that for any vertex $x, \sum_{i=1}^{|P|-1} e^{-p\left(x, u_{i}\right)}$ is $O\left(n^{3 / 4}\right)$. We partition the $u_{i}$ according to their hop-distance from $x$. Fix $j$ and consider all vertices in $P$ that are distance between $2^{j-1}$ and $2^{j}$ from $x$. By our claim above, there are at most $2^{j}$ such vertices. If $2^{j} \leq \sqrt{n}$, then we use the trivial bound of 1 ; otherwise, we use the bound $2^{2 j} / n^{3 / 2}$. So we have

$$
\begin{aligned}
\sum_{u_{i} \in P} e^{-p\left(x, u_{i}\right)} & =\sum_{j: 2^{j} \leq \sqrt{n}} O\left(2^{j}\right)+\sum_{j: 2^{j}>\sqrt{n}}\left(2^{j} * e^{-2^{2 j} / n^{3 / 2}}\right) \\
& =O\left(n^{1 / 2}\right)+O\left(n^{3 / 4}\right)=O\left(n^{3 / 4}\right) .
\end{aligned}
$$

Since sum of $d(x)$, over all $x$ in $V$, is $2 m$, we obtain the desired bound of $O\left(m n^{3 / 4}\right)$. 
THEOREM 5.8. The hitting and cover times for a 2-cobra walk on a graph with $n$ vertices are $O\left(m n^{3 / 4}\right)$ and $O\left(m n^{3 / 4} \log n\right)$, respectively.

Proof. For any start vertex $u$ and target vertex $v$, let $P$ be a shortest-hop path from $u$ to $v$, given by the sequence of vertices $u=u_{0}, u_{1}, u_{2}, \ldots, u_{|P|}=v$. By Lemma 5.2 and Equation (7) we have

$$
H(u, v) \leq H^{*}(u, v) \leq \sum_{i=0}^{|P|-1} d\left(u_{i}\right) R\left(u_{i}\right),
$$

which, by Lemma 5.7, is $O\left(m n^{3 / 4}\right)$. The bound on the cover time follows from Matthews' Theorem, Theorem 2.1.

\section{CONCLUSION}

We have derived several improved results for cobra walks. However, there remain many open problems, and corresponding gaps in our understanding. We have shown that for $d$-dimensional grids on $[0, n]^{d}$, the cover time is proportional to $n$, even for 2-cobra walks. The dependence on $d$, however, has not been determined, and more generally we would like to find a larger collection of graph types for which the cover time for 2-cobra walks is proportional to the diameter. Better results for general graphs are still open; we optimistically conjecture that for 2-cobra walks the worst case cover time on a graph with $n$ vertices is only $O(n \log n)$. (The star graph shows that it can be $\Omega(n \log n)$.) Perhaps most importantly, we believe there should be better methods available for analyzing cobra walks. Our results utilize techniques based on parallelism and bias, but we do not believe they are as yet taking full advantage of the power of cobra walks.

\section{REFERENCES}

[1] Micah Adler, Eran Halperin, Richard M. Karp, and Vijay V. Vazirani. 2003. A stochastic process on the hypercube with applications to peer-to-peer networks. In Proceedings of the 35th Annual ACM Symposium on Theory of Computing (STOC'03). ACM, New York, NY, 575-584. DOI : http://dx.doi.org/10.1145/780542.780626

[2] Noga Alon, Chen Avin, Michal Koucky, Gady Kozma, Zvi Lotker, and Mark R. Tuttle. 2008. Many random walks are faster than one. In Proceedings of the 20th Annual Symposium on Parallelism in Algorithms and Architectures (SPAA'08). ACM, New York, NY, 119-128. DOI : http://dx.doi.org/10.1145/1378533.1378557

[3] Siva R. Arthreya and Jan M. Swart. 2005. Branching-coalescing particle systems. Probab. Theor. Relat. Fields 131, 3 (2005), 376-414. DOI : http://dx.doi.org/10.1007/s00440-004-0377-4

[4] J. Augustine, G. Pandurangan, P. Robinson, S. Roche, and E. Upfal. 2015. Enabling robust and efficient distributed computation in dynamic peer-to-peer networks. In 2015 IEEE 56th Annual Symposium on Foundations of Computer Science (FOCS'15). 350-369. DOI : http://dx.doi.org/10.1109/FOCS.2015.29

[5] Yossi Azar, Andrei Z. Broder, Anna R. Karlin, Nathan Linial, and Steven Phillips. 1992. Biased random walks. In Proceedings of the 24th Annual ACM Symposium on Theory of Computing. ACM, 1-9.

[6] Itai Benjamini and Sebastian Müller. 2010. On the trace of branching random walks. arXiv:1002.2781 (2010).

[7] Petra Berenbrink, Colin Cooper, Robert Elsässer, Tomasz Radzik, and Thomas Sauerwald. 2010. Speeding up random walks with neighborhood exploration. In Proceedings of the 21st Annual ACM-SIAM Symposium on Discrete Algorithms (SODA'10). Society for Industrial and Applied Mathematics, Philadelphia, PA, 1422-1435. http://dl.acm.org/citation. cfm?id=1873601.1873716

[8] Andrei Broder. 1989. Generating random spanning trees. In Proceedings of the 30th Annual Symposium on Foundations of Computer Science, 1989. 442-447. DOI : http://dx.doi.org/10.1109/SFCS.1989.63516

[9] Jen-Yeu Chen and Gopal Pandurangan. 2012. Almost-optimal gossip-based aggregate computation. SIAM f. Comput. 41, 3 (2012), 455-483.

[10] F. Chung. 2005. Laplacians and the cheeger inequality for directed graphs. Ann. Comb. 9 (2005), 1-19.

[11] Colin Cooper, Robert Elsässer, Hirotaka Ono, and Tomasz Radzik. 2012. Coalescing random walks and voting on graphs. In Proceedings of the 2012 ACM Symposium on Principles of Distributed Computing (PODC'12). ACM, New York, NY, 47-56. DOI : http://dx.doi.org/10.1145/2332432.2332440

[12] Colin Cooper, Tomasz Radzik, and Nicolas Rivera. 2016. The coalescing-branching random walk on expanders and the dual epidemic process. In Proceedings of the 2016 ACM Symposium on Principles of Distributed Computing (PODC'16), George Giakkoupis (Ed.). ACM, 461-467. DOI : http://dx.doi.org/10.1145/2933057.2933119 
[13] Colin Cooper, Tomasz Radzik, and Nicolas Rivera. 2017. Improved cover time bounds for the coalescing-branching random walk on graphs. In Proceedings of the 2017 ACM Symposium on Parallelism in Algorithms and Architectures (SPAA'17). 305-312.

[14] Nedialko B. Dimitrov and C. Greg Plaxton. 2005. Optimal cover time for a graph-based coupon collector process. In Proceedings of the 32nd International Conference on Automata, Languages and Programming (ICALP'05). SpringerVerlag, Berlin, 702-716. DOI: http://dx.doi.org/10.1007/11523468_57

[15] Chinmoy Dutta, Gopal Pandurangan, Rajmohan Rajaraman, and Scott Roche. 2015. Coalescing-branching random walks on graphs. ACM Trans. Parallel Comput. 2, 3, Article 20 (Nov. 2015), 29 pages. DOI :http://dx.doi.org/10.1145/ 2817830

[16] Robert Elsasser and Thomas Sauerwald. 2011. Tight bounds for the cover time of multiple random walks. Theor. Comput. Sci. 412, 24 (2011), 2623-2641. Selected Papers from 36th International Colloquium on Automata, Languages and Programming (ICALP'09). DOI : http://dx.doi.org/10.1016/j.tcs.2010.08.010

[17] Uriel Feige. 1995. A tight upper bound on the cover time for random walks on graphs. Random Structures \& Algorithms 6, 1 (1995), 51-54.

[18] Uriel Feige. 1995. A tight lower bound on the cover time for random walks on graphs. Random Struct. Algorithms 6, 4 (July 1995), 433-438. DOI : http://dx.doi.org/10.1002/rsa.3240060406

[19] Uriel Feige, David Peleg, Prabhakar Raghavan, and Eli Upfal. 1990. Randomized broadcast in networks. Random Structures \& Algorithms 1, 4 (1990), 447-460.

[20] Ayalvadi J. Ganesh, Laurent Massouli, and Donald F. Towsley. 2005. The effect of network topology on the spread of epidemics. In INFOCOM (2006-10-04). IEEE, 1455-1466. http://dblp.uni-trier.de/db/conf/infocom/infocom2005. html\#GaneshMT05.

[21] Theodore E. Harris. 1963. The Theory of Branching Processes. Springer-Verlag, Berlin. xiv+230 pages.

[22] David A. Kessler. 2008. Epidemic size in the SIS model of endemic infections. f. Appl. Probab. 45, 3 (09 2008), 757-778. DOI : http://dx.doi.org/10.1239/jap/1222441828

[23] David Asher Levin, Yuval Peres, and Elizabeth Lee Wilmer. 2009. Markov Chains and Mixing Times. With a chapter on coupling from the past by James G. Propp and David B. Wilson. American Mathematical Society, Providence, R.I Retrieved from http://opac.inria.fr/record=b1128575

[24] Neal Madras and Rinaldo Schinazi. 1992. Branching random walks on trees. Stoch. Process. Appl. 42, 2 (1992), 255267. DOI : http://dx.doi.org/10.1016/0304-4149(92)90038-R

[25] M. Metropolis, A. Rosenbluth, M. Rosenbluth, A. Teller, and M. Teller. 1953. Equation of state calculations by fast computing machines. f. Chem. Phys. 21 (1953), 1087-1092.

[26] Michael Mitzenmacher and Eli Upfal. 2005. Probability and Computing: Randomized Algorithms and Probabilistic Analysis. Cambridge University Press.

[27] Sebastien Roch. 2015. Modern Discrete Probability: An Essential Toolkit. Class Lecture Notes. Retrieved from http:// www.math.wisc.edu/roch/mdp/index.html.

[28] Daniel Spielman. 2012. Lecture Notes in Spectral Graph Theory. Yale University.

[29] Rongfeng Sun and Jan M. Swart. 2008. The Brownian net. Ann. Probab. 36, 3 (2008), 1153-1208.

[30] Salil P. Vadhan. 2011. Pseudorandomness. Found. Trends Theor. Comput. Sci. 7, 1-3 (2011), 1-336. DOI : http://dx.doi. org/10.1561/0400000010

[31] Piet Van Mieghem. 2011. The N-intertwined SIS epidemic network model. Computing 93, 2-4 (Dec. 2011), 147-169. DOI : http://dx.doi.org/10.1007/s00607-011-0155-y

Received March 2017; revised October 2017; accepted January 2018 